\title{
A generalization of Kawanaka's identity for Hall-Littlewood polynomials and applications
}

\author{
Masao Ishikawa $\cdot$ Frédéric Jouhet $\cdot$ Jiang Zeng
}

Received: March 4, 2004 / Revised: June 14, 2005 / Accepted: November 4, 2005

(C) Springer Science + Business Media, LLC 2006

\begin{abstract}
An infinite summation formula of Hall-Littlewood polynomials due to Kawanaka is generalized to a finite summation formula, which implies, in particular, twelve more multiple $q$-identities of Rogers-Ramanujan type than those previously found by Stembridge and the last two authors.
\end{abstract}

Keywords Symmetric functions · Hall-Littlewood polynomials · $Q$-series · Rogers-Ramanujan type identities

\section{Introduction}

Recently, starting from two infinite summation formulae for Hall-Littlewood polynomials, two of the present authors [7] have generalized a method due to Macdonald [9] to obtain new finite summation formulae for these polynomials. This approach permits them to extend Stembridge's list of multiple $q$-series identities of Rogers-Ramanujan type [12]. Conversely these symmetric functions identities can be viewed as a generalization of RogersRamanujan identities. In view of the numerous formulae of Rogers-Ramanujan type [11] one may speculate that there should be more such generalizations starting from other infinite summation formulae for Hall-Littlewood polynomials. However, as pointed out in [7], when one passes from an infinite summation to a finite summation, one may need to modify the coefficients normalizing Hall-Littlewood polynomials in order to obtain some useful formulae.

\footnotetext{
M. Ishikawa $(\bowtie)$

Department of Mathematics, Faculty of Education Tottori University, Tottori 680-8551, Japan e-mail: ishikawa@fed.tottori-u.ac.jp

F. Jouhet · J. Zeng

Institut Camille Jordan, Université Claude Bernard (Lyon I) 43, bd du 11 Novembre 1918, 69622 Villeurbanne Cedex, France

e-mail: \{jouhet, zeng\}@math.univ-lyon1.fr
} 
In this paper we take up on Kawanaka's new infinite summation identities of HallLittlewood polynomials [8] and show that one of his formulae has a finite summation generalization.

We first need to recall some standard notations of $q$-series, which can be found in [5]. Set $(x)_{0}:=(x ; q)_{0}=1$ and for $n \geq 1$

$$
(x)_{n}:=(x ; q)_{n}=\prod_{k=0}^{n}\left(1-x q^{k}\right), \quad(x)_{\infty}:=(x ; q)_{\infty}=\prod_{k=0}^{\infty}\left(1-x q^{k}\right) .
$$

For $n \geq 0$ and $r \geq 1$, set

$$
\left(a_{1}, \ldots, a_{r} ; q\right)_{n}=\prod_{i=1}^{r}\left(a_{i}\right)_{n} \quad\left(a_{1}, \ldots, a_{r} ; q\right)_{\infty}=\prod_{i=1}^{r}\left(a_{i}\right)_{\infty} .
$$

The $q$-binomial identity [1] then reads as follows:

$$
\sum_{m \geq 0} \frac{(a)_{m}}{(q)_{m}} x^{m}=\frac{(a x)_{\infty}}{(x)_{\infty}}
$$

which reduces to the finite $q$-binomial identity by substitution $a \rightarrow q^{-n}$ and $x \rightarrow q^{n} x$ :

$$
(x)_{n}=\sum_{m \geq 0}(-1)^{m} q^{\left(\begin{array}{c}
m \\
2
\end{array}\right)} \frac{(q)_{n}}{(q)_{m}(q)_{n-m}} x^{m}
$$

and to the following identity of Euler when $a=0$ :

$$
\frac{1}{(x)_{\infty}}=\sum_{m \geq 0} \frac{x^{m}}{(q)_{m}}
$$

Let $n \geq 1$ be a fixed integer and $S_{n}$ denote the group of permutations of the set $\{1,2, \ldots, n\}$. Let $X=\left\{x_{1}, \ldots, x_{n}\right\}$ be a set of indeterminates and $q$ a parameter. For each partition $\lambda=\left(\lambda_{1}, \ldots, \lambda_{n}\right)$ of length $\leq n$, if $m_{i}:=m_{i}(\lambda)$ is the multiplicity of part $i$ in $\lambda$, then we also denote $\lambda$ by $\left(1^{m_{1}} 2^{m_{2}} \ldots\right)$. Recall that the Hall-Littlewood polynomials $P_{\lambda}(X, q)$ are defined by $[9$, p. 208]:

$$
P_{\lambda}(X, q)=\prod_{i \geq 1} \frac{(1-q)^{m_{i}}}{(q)_{m_{i}}} \sum_{w \in S_{n}} w\left(x_{1}^{\lambda_{1}} \ldots x_{n}^{\lambda_{n}} \prod_{i<j} \frac{x_{i}-q x_{j}}{x_{i}-x_{j}}\right) .
$$

Since ( [9, p. 207])

$$
\sum_{w \in S_{n}} w\left(\prod_{i<j} \frac{x_{i}-q x_{j}}{x_{i}-x_{j}}\right)=\frac{(q)_{n}}{(1-q)^{n}}
$$


we see that the coefficient of $x_{1}^{\lambda_{1}} \ldots x_{n}^{\lambda_{n}}$ in $P_{\lambda}$ is 1 . Set

$$
\Phi(X):=\prod_{i} \frac{1+q x_{i}}{1-x_{i}} \prod_{j<k} \frac{1-q^{2} x_{j} x_{k}}{1-x_{j} x_{k}} .
$$

Our starting point is the following result due to Kawanaka [8]:

$$
\sum_{\lambda}\left(\prod_{i \geq 1}(-q)_{m_{i}}\right) P_{\lambda}\left(X, q^{2}\right)=\Phi(X) .
$$

Since Kawanaka's proof uses the representation theory of groups we shall give another proof using Pieri's rule for Hall-Littlewood polynomials.

For each sequence $\xi \in\{ \pm 1\}^{n}$, set $X^{\xi}:=\left\{x_{1}^{\xi_{1}}, \ldots, x_{n}^{\xi_{n}}\right\}$. Our finite extension of Kawanaka's formula then reads as follows:

Theorem 1. For $k \geq 1$ the following identity holds

$$
\sum_{\lambda_{1} \leq k}\left(\prod_{i=1}^{k-1}(-q)_{m_{i}}\right) P_{\lambda}\left(X, q^{2}\right)=\sum_{\xi \in\{ \pm 1\}^{n}} \Phi\left(X^{\xi}\right) \prod_{i} x_{i}^{k\left(1-\xi_{i}\right) / 2} .
$$

Remark. In the case $q=0$, the right-hand side of (5) can be written as a quotient of determinants and the formula reduces to a known identity of Schur functions [6, p. 85].

For any partition $\lambda$ it will be convenient to adopt the following notation:

$$
(x)_{\lambda}:=(x ; q)_{\lambda}=(x)_{\lambda_{1}-\lambda_{2}}(x)_{\lambda_{2}-\lambda_{3}} \cdots
$$

Note that this is not the standard notation for $(x)_{\lambda}$ and corresponds to $b_{\lambda^{\prime}}(q)$ in $[9$, p. 210].

We also introduce the following generalization of $q$-binomial coefficients

$$
\left[\frac{n}{\lambda}\right]=\left[\frac{n}{\lambda}\right]_{q}:=\frac{(q)_{n}}{(q)_{n-\lambda_{1}}(q)_{\lambda}},
$$

with the convention that $\left[\begin{array}{l}n \\ \lambda\end{array}\right]=0$ if $\lambda_{1}>n$. If $\lambda=\left(\lambda_{1}\right)$ we recover the classical $q$-binomial coefficient. Finally, for any partition $\lambda$ we denote by $l(\lambda)$ the length of $\lambda$, i.e., the number of its positive parts, and define $n(\lambda):=\sum_{i}\left(\begin{array}{c}\lambda_{i} \\ 2\end{array}\right)$ and $n_{2}(\lambda)=\sum_{i} \lambda_{i}^{2}$. When $x_{i}=z q^{2 i-2}$ for $i \geq 1$ and then $z$ is replaced by $z q$, formula (5) specializes to the following identity.

Corollary 1. For $k \geq 1$ holds

$$
\begin{aligned}
& \sum_{l(\lambda) \leq k}\left(\prod_{i=1}^{k-1}(-q)_{\lambda_{i}-\lambda_{i+1}}\right) z^{|\lambda|} q^{n_{2}(\lambda)}\left[\begin{array}{l}
n \\
\lambda
\end{array}\right]_{q^{2}} \\
& \quad=\sum_{r=0}^{n}(-1)^{r} z^{k r} q^{(k+1) r^{2}}\left[\begin{array}{l}
n \\
r
\end{array}\right]_{q^{2}} \frac{(-z)_{2 n+1}}{\left(z^{2} q^{2 r} ; q^{2}\right)_{n+1}}\left(1-z q^{2 r}\right) .
\end{aligned}
$$


Now, as in $[7,12]$, we can prove the following key $q$-identity which allows to produce identities of Rogers-Ramanujan type:

Theorem 2. For $k \geq 1$,

$$
\begin{aligned}
& \sum_{l(\lambda) \leq k} z^{|\lambda|} q^{2 n(\lambda)} \frac{\left(a, b ; q^{-1}\right)_{\lambda_{1}}}{(-q)_{\lambda_{k}}(q)_{\lambda}} \\
& \quad=\frac{(-z / q)_{\infty}}{(a b z)_{\infty}} \sum_{r \geq 0}(-1)^{r} z^{k r} q^{r+(2 k+2)\left(\begin{array}{l}
r \\
2
\end{array}\right)} \frac{\left(a, b ; q^{-1}\right)_{r}}{\left(q^{2} ; q^{2}\right)_{r}} \frac{\left(a z q^{r}, b z q^{r}\right)_{\infty}}{\left(z^{2} q^{2 r-2} ; q^{2}\right)_{\infty}}\left(1-z q^{2 r-1}\right) .
\end{aligned}
$$

This paper is organized as follows. In Section 2 we give a new proof of Kawanaka's formula using Pieri's rule for Hall-Littlewood polynomials since Kawanaka's original proof uses the representation theory of groups. In Section 3, we derive from Theorem 2 twelve multiple analogs of Rogers-Ramanujan type identities. In Section 4 we give the proofs of Theorem 1 and Corollary 1, and some consequences, and defer the elementary proof, i.e., without using the Hall-Littlewood polynomials, of Theorem 2, Corollary 1 and other multiple $q$-series identities to Section 5. To prove Theorems 1 and 2 we apply the generating function technique which was developed in $[7,9,12]$.

\section{Another proof of Kawanaka's identity}

Recall [9, p. 230, Ex. 1] the following summation of Hall-Littlewood polynomials:

$$
\sum_{\mu} P_{\mu}(X, q)=\prod_{i} \frac{1}{1-x_{i}} \prod_{i<j} \frac{1-q x_{i} x_{j}}{1-x_{i} x_{j}} .
$$

By replacing $q$ by $q^{2}$, we get

$$
\sum_{\mu} P_{\mu}\left(X, q^{2}\right)=\prod_{i} \frac{1}{1-x_{i}} \prod_{i<j} \frac{1-q^{2} x_{i} x_{j}}{1-x_{i} x_{j}} .
$$

Note that

$$
\sum_{r \geq 0} e_{k}(X) q^{k}=\prod_{i}\left(1+q x_{i}\right)
$$

where $e_{r}(X)$ stands for the $r$-th elementary symmetric function. Identities (8) and (9) imply

$$
\sum_{\mu} \sum_{r} q^{r} P_{\mu}\left(X, q^{2}\right) e_{r}(X)=\prod_{i} \frac{1+q x_{i}}{1-x_{i}} \prod_{i<j} \frac{1-q^{2} x_{i} x_{j}}{1-x_{i} x_{j}} .
$$

From [9, p. 209, (2.8)], we have

$$
P_{\left(1^{r}\right)}(X, q)=e_{r}(X)
$$


and this shows that

$$
\sum_{\mu} \sum_{r} q^{r} P_{\mu}\left(X, q^{2}\right) P_{\left(1^{r}\right)}\left(X, q^{2}\right)=\prod_{i} \frac{1+q x_{i}}{1-x_{i}} \prod_{i<j} \frac{1-q^{2} x_{i} x_{j}}{1-x_{i} x_{j}} .
$$

Let $f_{\mu \nu}^{\lambda}(q)$ be the coefficients defined by

$$
P_{\mu}(X, q) P_{\nu}(X, q)=\sum_{\lambda} f_{\mu \nu}^{\lambda}(q) P_{\lambda}(X, q),
$$

then, by $[9$, p. $215(3.2)]$ we have

$$
f_{\mu\left(1^{m}\right)}^{\lambda}(q)=\prod_{i \geq 1}\left[\begin{array}{c}
\lambda_{i}^{\prime}-\lambda_{i+1}^{\prime} \\
\lambda_{i}^{\prime}-\mu_{i}^{\prime}
\end{array}\right]
$$

(and therefore $f_{\mu\left(1^{m}\right)}^{\lambda}(q)=0$ unless $\lambda \backslash \mu$ is a vertical $m$-strip, or $m$-vs, which means $\lambda \subset \mu$, $|\lambda \backslash \mu|=m$ and there is at most one cell in each row of the Ferrers diagram of $\lambda \backslash \mu$ ). Thus we have

$$
\begin{gathered}
\sum_{\lambda} \sum_{\substack{\mu \\
\lambda \backslash \mu \mathrm{vs}}} q^{|\lambda-\mu|} \prod_{i \geq 1}\left[\begin{array}{c}
\lambda_{i}^{\prime}-\lambda_{i+1}^{\prime} \\
\lambda_{i}^{\prime}-\mu_{i}^{\prime}
\end{array}\right]_{q^{2}} P_{\lambda}\left(X, q^{2}\right) \\
=\prod_{i} \frac{1+q x_{i}}{1-x_{i}} \prod_{i<j} \frac{1-q^{2} x_{i} x_{j}}{1-x_{i} x_{j}} .
\end{gathered}
$$

Applying the identity (see [1], and [13] for a bijective proof):

$$
\sum_{k=0}^{n} q^{k}\left[\begin{array}{l}
n \\
k
\end{array}\right]_{q^{2}}=\prod_{k=1}^{n}\left(1+q^{k}\right)
$$

we conclude that

$$
\sum_{\substack{\mu \\
\lambda \backslash \mu \mathrm{vs}}} q^{|\lambda-\mu|} \prod_{i \geq 1}\left[\begin{array}{c}
\lambda_{i}^{\prime}-\lambda_{i+1}^{\prime} \\
\lambda_{i}^{\prime}-\mu_{i}^{\prime}
\end{array}\right]_{q^{2}}=\prod_{i \geq 1} \prod_{k=1}^{\lambda_{i}^{\prime}-\lambda_{i+1}^{\prime}}\left(1+q^{k}\right)
$$

which is precisely what we desired to prove.

Remark. For a node $v=(i, j)$ in the diagram of $\lambda$, the arm-length $a(v)$ and the leg-length $l(v)$ of $\lambda$ at $v$ are defined by $a(v)=\lambda_{i}-j$ and $l(v)=\lambda_{j}^{\prime}-i$ respectively. Kawanaka [8, (5.2)] proved another identity for Hall-Littlewood polynomials:

$$
\sum_{\lambda} q^{o(\lambda) / 2}\left(\prod_{\substack{v \in \lambda, a(v)=0 \\ l(v) \text { even }}}\left(1-q^{l(v)+1}\right)\right) P_{\lambda}(X, q)=\prod_{i \leq j} \frac{1-q x_{i} x_{j}}{1-x_{i} x_{j}},
$$

where the sum on the left is taken over all partitions $\lambda$ such that $m_{i}(\lambda)$ is even for odd $i$ and

$$
o(\lambda)=\sum_{i \text { odd }} m_{i}(\lambda)
$$

It would be possible to prove this identity in the same manner as above. 
There is a related identity about Hall-Littlewood polynomials in Macdonald's book [9, p. 219]:

$$
\sum_{\lambda} q^{n(\lambda)}\left(\prod_{j=1}^{l(\lambda)}\left(1+q^{1-j} y\right)\right) P_{\lambda}(X, q)=\prod_{i \geq 1} \frac{1+x_{i} y}{1-x_{i}} .
$$

\section{Multiple identities of Rogers-Ramanujan type}

We shall derive several identities of Rogers-Ramanujan type from Theorem 2. First we note that if $z=q^{2}$ identity (7) reduces to

$$
\begin{aligned}
& \sum_{l(\lambda) \leq k} q^{|\lambda|+n_{2}(\lambda)} \frac{\left(a, b ; q^{-1}\right)_{\lambda_{1}}}{(-q)_{\lambda_{k}}(q) \lambda}=\frac{1}{\left(q, a b q^{2}\right)_{\infty}} \\
& \quad \times \sum_{r \geq 0}(-1)^{r} q^{(2 k+1) r+(2 k+2)\left(\begin{array}{l}
r \\
2
\end{array}\right)\left(a, b ; q^{-1}\right)_{r}\left(a q^{r+2}, b q^{r+2}\right)_{\infty}\left(1-q^{2 r+1}\right),}
\end{aligned}
$$

and if $z=q$ it becomes

$$
\begin{aligned}
& \sum_{l(\lambda) \leq k} q^{n_{2}(\lambda)} \frac{\left(a, b ; q^{-1}\right)_{\lambda_{1}}}{(-q)_{\lambda_{k}}(q)_{\lambda}}=\frac{1}{(q, a b q)_{\infty}} \\
& \quad \times\left((a q, b q)_{\infty}+2 \sum_{r \geq 1}(-1)^{r} q^{(k+1) r^{2}}\left(a, b ; q^{-1}\right)_{r}\left(a q^{r+1}, b q^{r+1}\right)_{\infty}\right) .
\end{aligned}
$$

We need the following two forms of Jacobi triple product identity [1, p. 21]:

$$
\begin{aligned}
J(x, q):=(q, x, q / x)_{\infty} & =\sum_{r=0}^{\infty}(-1)^{r} x^{r} q^{\left(\begin{array}{c}
r \\
2
\end{array}\right)}\left(1-q^{2 r+1} / x^{2 r+1}\right) \\
& =1+\sum_{r=1}^{\infty}(-1)^{r} x^{r} q^{\left(\begin{array}{c}
r \\
2
\end{array}\right)}\left(1+q^{r} / x^{2 r}\right) .
\end{aligned}
$$

Theorem 3. For $k \geq 1$, the following identities hold

$$
\begin{gathered}
\sum_{l(\lambda) \leq k} \frac{q^{|\lambda|+n_{2}(\lambda)}}{(-q)_{\lambda_{k}}(q)_{\lambda}}=\frac{\left(q^{2 k+2}, q^{2 k+1}, q ; q^{2 k+2}\right)_{\infty}}{(q)_{\infty}}, \\
\sum_{l(\lambda) \leq k} \frac{q^{|\lambda|+n_{2}(\lambda)-\left(\lambda_{1}^{2}+\lambda_{1}\right) / 2}(-q)_{\lambda_{1}}}{(-q)_{\lambda_{k}}(q)_{\lambda}}=\frac{(-q)_{\infty}}{(q)_{\infty}}\left(q^{2 k+1}, q^{2 k}, q ; q^{2 k+1}\right)_{\infty}, \\
\sum_{l(\lambda) \leq k} \frac{q^{2|\lambda|+2 n_{2}(\lambda)-\lambda_{1}^{2}}\left(-q ; q^{2}\right)_{\lambda_{1}}}{\left(-q^{2} ; q^{2}\right)_{\lambda_{k}}\left(q^{2} ; q^{2}\right)_{\lambda}}=\frac{\left(-q ; q^{2}\right)_{\infty}}{\left(q^{2} ; q^{2}\right)_{\infty}}\left(q^{4 k+2}, q^{4 k+1}, q ; q^{4 k+2}\right)_{\infty}, \\
\sum_{l(\lambda) \leq k} \frac{q^{2|\lambda|+2 n_{2}(\lambda)-2 \lambda_{1}^{2}-\lambda_{1}}(-q)_{2 \lambda_{1}}}{\left(-q^{2} ; q^{2}\right)_{\lambda_{k}}\left(q^{2} ; q^{2}\right)_{\lambda}}=\frac{(-q)_{\infty}}{(q)_{\infty}}\left(q^{4 k}, q^{4 k-1}, q ; q^{4 k}\right)_{\infty},
\end{gathered}
$$




$$
\begin{aligned}
\sum_{l(\lambda) \leq k} \frac{q^{n_{2}(\lambda)}}{(-q)_{\lambda_{k}}(q)_{\lambda}} & =\frac{\left(q^{2 k+2}, q^{k+1}, q^{k+1} ; q^{2 k+2}\right)_{\infty}}{(q)_{\infty}}, \\
\sum_{l(\lambda) \leq k} \frac{q^{n_{2}(\lambda)-\left(\lambda_{1}^{2}+\lambda_{1}\right) / 2}(-q)_{\lambda_{1}}}{(-q)_{\lambda_{k}}(q)_{\lambda}} & =\frac{(-1)_{\infty}}{(q)_{\infty}}\left(q^{2 k+1}, q^{k}, q^{k+1} ; q^{2 k+1}\right)_{\infty}, \\
\sum_{l(\lambda) \leq k} \frac{q^{2 n_{2}(\lambda)-\lambda_{1}^{2}}\left(-q ; q^{2}\right)_{\lambda_{1}}}{\left(-q^{2} ; q^{2}\right)_{\lambda_{k}}\left(q^{2} ; q^{2}\right)_{\lambda}} & =\frac{\left(-q ; q^{2}\right)_{\infty}}{\left(q^{2} ; q^{2}\right)_{\infty}}\left(q^{4 k+2}, q^{2 k+1}, q^{2 k+1} ; q^{4 k+2}\right)_{\infty} .
\end{aligned}
$$

Proof: For identities (17)-(20), first set $(a, b)=(0,0),\left(-q^{-1}, 0\right),\left(-q^{-1 / 2}, 0\right)$ and $\left(-q^{-1 / 2},-q^{-1}\right)$ in (13), respectively, and then apply (15).

For identities $(21)-(23)$, first set $(a, b)=(0,0),\left(-q^{-1 / 2}, 0\right)$ and $\left(-q^{-1}, 0\right)$ in (14), respectively, and then apply (16).

Note that, for (19), (20) and (23), we need to replace $q$ by $q^{2}$ at last.

Theorem 4. For $k \geq 1$, the following identities hold

$$
\begin{aligned}
\sum_{l(\lambda) \leq k} \frac{q^{|\lambda|+n_{2}(\lambda)-\left(\lambda_{1}^{2}+3 \lambda_{1}\right) / 2}(-q)_{\lambda_{1}}\left(1-q^{\lambda_{1}}\right)}{(-q)_{\lambda_{k}}(q)_{\lambda}} & =\frac{(-q)_{\infty}}{(q)_{\infty}}\left(q^{2 k+1}, q^{2 k-1}, q^{2} ; q^{2 k+1}\right)_{\infty}, \\
\sum_{l(\lambda) \leq k} \frac{q^{2|\lambda|+2 n_{2}(\lambda)-\lambda_{1}^{2}-2 \lambda_{1}}\left(-q ; q^{2}\right)_{\lambda_{1}}}{\left(-q^{2} ; q^{2}\right)_{\lambda_{k}}\left(q^{2} ; q^{2}\right)_{\lambda}} & =\frac{\left(-q ; q^{2}\right)_{\infty}}{\left(q^{2} ; q^{2}\right)_{\infty}}\left(q^{4 k+2}, q^{4 k-1}, q^{3} ; q^{4 k+2}\right)_{\infty}, \\
\sum_{l(\lambda) \leq k} \frac{q^{|\lambda|+n_{2}(\lambda)-\lambda_{1}}}{(-q)_{\lambda_{k}}(q)_{\lambda}} & =\frac{\left(q^{2 k+2}, q^{2 k}, q^{2} ; q^{2 k+2}\right)_{\infty}}{(q)_{\infty}} \\
\sum_{l(\lambda) \leq k} \frac{q^{|\lambda|+n_{2}(\lambda)-2 \lambda_{1}}\left(1-q^{2 \lambda_{1}}\right)}{(-q)_{\lambda_{k}}(q)_{\lambda}} & =\frac{\left(q^{2 k+2}, q^{2 k-1}, q^{3} ; q^{2 k+2}\right)_{\infty}}{(q)_{\infty}} \\
\sum_{l(\lambda) \leq k} \frac{q^{n_{2}(\lambda)-\lambda_{1}}}{(-q)_{\lambda_{k}}(q)_{\lambda}} & =\frac{(-1)_{\infty}}{\left(q^{2} ; q^{2}\right)_{\infty}}\left(q^{2 k+2}, q^{k}, q^{k+2} ; q^{2 k+2}\right)_{\infty} .
\end{aligned}
$$

Proof: For $i \in\{0,1,2\}$, denote by $\left[b^{i}\right]$ the operation of extracting the coefficient of $b^{i}$ in the corresponding identity. For (24)-(27), apply the following operations to (13) respectively: $a=-q^{-1}$ and $(1-1 / q)[b], a=0$ and $\left[b^{0}\right]+(1-1 / q)[b], a=-q^{-1 / 2}$ and $\left[b^{0}\right]+(1-$ $1 / q)[b], a=0$ and $[b]+(1-1 / q)\left[b^{2}\right]$, and then apply (15). Note that, for $(25)$, we need to replace $q$ by $q^{2}$ at last.

For (28) apply the operations $a=0$ and $(1-1 / q)[b]$ to (14) and then apply (16).

Remark. As speculated by the anonymous referee, all of the Rogers-Ramanujan type identities given in Theorems 3 and 4 are known.

For example, specializing equation (3.4) of Bressoud [4] (see also [3]) with $k \rightarrow k+1$ and $r=1, r=2$ and $r=k+1$, respectively, we recover identities (17), (26)) and (21); while specializing equation (3.9) of Bressoud [4] with $k \rightarrow k+1$ and $r=1, r=2$ and $r=k+1$, respectively, we recover (19), (25) and (23). 
Since we derived all these identities in Theorems 3 and 4 from the two master identities (13) and (14), instead of identifying each identity individually, it suffices to identify the latter two with known results in the literature. In 1984, by means of Bailey chains, Andrews proved a remarkable generalization of Bailey's lemma [2, Thm. 1], which contains many multiple Rogers-Ramanujan type identities as special cases. In particular, identities (13) and (14) are limit cases of Andrews' theorem. More precisely, to derive (13), set $a=q, b_{k}=1 / a$, $c_{k}=1 / b$ in Andrews' formula and let $N, b_{1}, c_{1}, \ldots, b_{k-1}, c_{k-1} \rightarrow \infty$, finally apply the Bailey pair E(3) of Slater's paper [10]. To derive (14) we do the same thing except that we set $a=1$ and apply the Bailey pair B(3) of Slater's paper [10].

When $k=1$, identities (17), (18), (20), (27) and (23) reduce directly to special cases of the $q$-binomial identity (1). For example, when $k=1$ identity (20) reduces to

$$
\sum_{n=0}^{\infty} \frac{q^{n}\left(-q ; q^{2}\right)_{n}}{\left(q^{2} ; q^{2}\right)_{n}} \frac{\left(-q^{2} ; q^{2}\right)_{\infty}}{\left(q ; q^{2}\right)_{\infty}}
$$

which is the $q$-binomial identity (1) after substitutions $q \rightarrow q^{2}, a \rightarrow-q$ and $x \rightarrow q$. The other identities reduce to the following Rogers-Ramanujan type identities:

$$
\begin{aligned}
\sum_{n=0}^{\infty} \frac{q^{n^{2}+2 n}\left(-q ; q^{2}\right)_{n}}{\left(q^{4} ; q^{4}\right)_{n}} & =\frac{\left(-q ; q^{2}\right)_{\infty}}{\left(q^{2} ; q^{2}\right)_{\infty}}\left(q, q^{5}, q^{6} ; q^{6}\right)_{\infty}, \\
\sum_{n=0}^{\infty} \frac{q^{n^{2}}}{\left(q^{2} ; q^{2}\right)_{n}} & =\frac{\left(q^{2}, q^{2}, q^{4} ; q^{4}\right)_{\infty}}{(q)_{\infty}}, \\
\sum_{n=0}^{\infty} \frac{q^{n^{2}}\left(-q ; q^{2}\right)_{n}}{\left(q^{4} ; q^{4}\right)_{n}} & =\frac{\left(-q ; q^{2}\right)_{\infty}}{\left(q^{2} ; q^{2}\right)_{\infty}}\left(q^{3}, q^{3}, q^{6} ; q^{6}\right)_{\infty}
\end{aligned}
$$

Note that (30) is again a special case of the $q$-binomial identity (1) and (31) is (25) of Slater's list [11].

\section{Proof of Theorem 1 and consequences}

\subsection{Proof of Theorem 1}

For any statement $A$ it will be convenient to introduce the Boolean function $\chi(A)$, which is 1 if $A$ is true and 0 if $A$ is false. Consider the generating function

$$
S(u)=\sum_{\lambda_{0}, \lambda}\left(\prod_{i=1}^{\lambda_{0}-1}(-q)_{m_{i}}\right) P_{\lambda}\left(X, q^{2}\right) u^{\lambda_{0}}
$$

where the sum is over all partitions $\lambda=\left(\lambda_{1}, \ldots, \lambda_{n}\right)$ and the integers $\lambda_{0} \geq \lambda_{1}$. Suppose $\lambda=\left(\mu_{1}^{r_{1}} \mu_{2}^{r_{2}} \ldots \mu_{k}^{r_{k}}\right)$, where $\mu_{1}>\mu_{2}>\cdots>\mu_{k} \geq 0$ and $\left(r_{1}, \ldots, r_{k}\right)$ is a composition of $n$.

Let $S_{n}^{\lambda}$ be the set of permutations of $S_{n}$ which fix $\lambda$. Each $w \in S_{n} / S_{n}^{\lambda}$ corresponds to a surjective mapping $f: X \longrightarrow\{1,2, \ldots, k\}$ such that $\left|f^{-1}(i)\right|=r_{i}$. For any subset $Y$ of $X$, let $p(Y)$ denote the product of the elements of $Y$ (in particular, $p(\emptyset)=1$ ). We can rewrite $\underline{\text { Springer }}$ 
Hall-Littlewood functions as follows:

$$
P_{\lambda}\left(X, q^{2}\right)=\sum_{f} p\left(f^{-1}(1)\right)^{\mu_{1}} \cdots p\left(f^{-1}(k)\right)^{\mu_{k}} \prod_{f\left(x_{i}\right)<f\left(x_{j}\right)} \frac{x_{i}-q^{2} x_{j}}{x_{i}-x_{j}},
$$

summed over all surjective mappings $f: X \longrightarrow\{1,2, \ldots, k\}$ such that $\left|f^{-1}(i)\right|=r_{i}$. Furthermore, each such $f$ determines a filtration of $X$ :

$$
\mathcal{F}: \quad \emptyset=F_{0} \subsetneq F_{1} \subsetneq \cdots \subsetneq F_{k}=X,
$$

according to the rule $x_{i} \in F_{l} \Longleftrightarrow f\left(x_{i}\right) \leq l$ for $1 \leq l \leq k$. Conversely, such a filtration $\mathcal{F}=\left(F_{0}, F_{1}, \ldots, F_{k}\right)$ determines a surjection $f: X \longrightarrow\{1,2, \ldots, k\}$ uniquely. Thus we can write:

$$
P_{\lambda}\left(X, q^{2}\right)=\sum_{\mathcal{F}} \pi_{\mathcal{F}} \prod_{1 \leq i \leq k} p\left(F_{i} \backslash F_{i-1}\right)^{\mu_{i}}
$$

summed over all the filtrations $\mathcal{F}$ such that $\left|F_{i}\right|=r_{1}+r_{2}+\cdots+r_{i}$ for $1 \leq i \leq k$, and

$$
\pi_{\mathcal{F}}=\prod_{f\left(x_{i}\right)<f\left(x_{j}\right)} \frac{x_{i}-q^{2} x_{j}}{x_{i}-x_{j}},
$$

where $f$ is the function defined by $\mathcal{F}$.

Now let $v_{i}=\mu_{i}-\mu_{i+1}$ if $1 \leq i \leq k-1$ and $v_{k}=\mu_{k}$, thus $v_{i}>0$ if $i<k$ and $v_{k} \geq 0$. Furthermore, let $\mu_{0}=\lambda_{0}$ and $v_{0}=\mu_{0}-\mu_{1}$ in the definition of $S(u)$, so that $v_{0} \geq 0$ and $\mu_{0}=v_{0}+v_{1}+\cdots+v_{k}$. Define $c_{\mathcal{F}}=\prod_{i=1}^{k}(-q)_{\left|F_{i} \backslash F_{i-1}\right|}$ for any filtration $\mathcal{F}$. Thus, since the lengths of columns of $\lambda$ are $\left|F_{j}\right|=r_{1}+\cdots+r_{j}$ with multiplicities $v_{j}$ and $r_{j}=m_{\mu_{j}}(\lambda)$ for $1 \leq j \leq k$, we have

$$
\begin{aligned}
\prod_{i=1}^{\lambda_{0}-1}(-q)_{m_{i}}=c_{\mathcal{F}} \times & \left(\chi\left(v_{k}=0\right)(-q)_{\left|F_{k} \backslash F_{k-1}\right|}+\chi\left(v_{k} \neq 0\right)\right)^{-1} \\
& \times\left(\chi\left(v_{0}=0\right)(-q)_{\left|F_{1}\right|}+\chi\left(v_{0} \neq 0\right)\right)^{-1} .
\end{aligned}
$$

Let $F(X)$ be the set of filtrations of $X$. Summarizing we obtain

$$
\begin{aligned}
S(u)= & \sum_{\mathcal{F} \in F(X)} c_{\mathcal{F}} \pi_{\mathcal{F}} \sum_{v_{1}>0}\left(u p\left(F_{1}\right)\right)^{\nu_{1}} \cdots \sum_{v_{k-1}>0}\left(u p\left(F_{k-1}\right)\right)^{v_{k-1}} \\
& \times \sum_{\nu_{0} \geq 0} \frac{u^{\nu_{0}}}{\chi\left(v_{0}=0\right)(-q)_{\left|F_{1}\right|}+\chi\left(\nu_{0} \neq 0\right)} \\
& \times \sum_{\nu_{k} \geq 0} \frac{u^{\nu_{k}} p\left(F_{k}\right)^{\nu_{k}}}{\chi\left(v_{k}=0\right)(-q)_{\left|F_{k} \backslash F_{k-1}\right|}+\chi\left(v_{k} \neq 0\right)} .
\end{aligned}
$$

For any filtration $\mathcal{F}$ of $X$ set

$$
\mathcal{A}_{\mathcal{F}}(X, u)=c_{\mathcal{F}} \prod_{j}\left[\frac{p\left(F_{j}\right) u}{1-p\left(F_{j}\right) u}+\frac{\chi\left(F_{j}=X\right)}{(-q)_{\left|F_{j} \backslash F_{j-1}\right|}}+\frac{\chi\left(F_{j}=\emptyset\right)}{(-q)_{\left|F_{1}\right|}}\right] .
$$


It follows from (34) that

$$
S(u)=\sum_{\mathcal{F} \in F(X)} \pi_{\mathcal{F}} \mathcal{A}_{\mathcal{F}}(X, u) .
$$

Hence $S(u)$ is a rational function of $u$ with simple poles at $1 / p(Y)$, where $Y$ is a subset of $X$. We are now proceeding to compute the corresponding residue $c(Y)$ at each pole $u=1 / p(Y)$.

Let us start with $c(\emptyset)$. Writing $\lambda_{0}=\lambda_{1}+k$ with $k \geq 0$, we see that

$$
\begin{aligned}
S(u) & =\sum_{\lambda} f_{\lambda}(q) P_{\lambda}\left(X, q^{2}\right) u^{\lambda_{1}} \sum_{k \geq 0} \frac{u^{k}}{\chi(k=0)(-q)_{m_{\lambda_{1}}}+\chi(k \neq 0)} \\
& =\sum_{\lambda} f_{\lambda}(q) P_{\lambda}\left(X, q^{2}\right) u^{\lambda_{1}}\left(\frac{u}{1-u}+\frac{1}{(-q)_{m_{\lambda_{1}}}}\right) .
\end{aligned}
$$

It follows from (4) that

$$
c(\emptyset)=[S(u)(1-u)]_{u=1}=\Phi(X) .
$$

For the computations of other residues, we need some more notation. For any $Y \subseteq X$, let $Y^{\prime}=X \backslash Y$ and $-Y=\left\{x_{i}^{-1}: x_{i} \in Y\right\}$. Then

$$
c(Y)=\left[\sum_{\mathcal{F}} \pi_{\mathcal{F}} \mathcal{A}_{\mathcal{F}}(X, u)(1-p(Y) u)\right]_{u=p(-Y)} .
$$

If $Y \notin \mathcal{F}$, the corresponding summand is equal to 0 . Thus we need only to consider the following filtrations $\mathcal{F}$ :

$$
\emptyset=F_{0} \subsetneq \cdots \subsetneq F_{t}=Y \subsetneq \cdots \subsetneq F_{k}=X \quad 1 \leq t \leq k .
$$

We may then split $\mathcal{F}$ into two filtrations $\mathcal{F}_{1}$ and $\mathcal{F}_{2}$ :

$$
\begin{aligned}
& \mathcal{F}_{1}: \emptyset \subsetneq-\left(Y \backslash F_{t-1}\right) \subsetneq \cdots \subsetneq-\left(Y \backslash F_{1}\right) \subsetneq-Y, \\
& \mathcal{F}_{2}: \emptyset \subsetneq F_{t+1} \backslash Y \subsetneq \cdots \subsetneq F_{k-1} \backslash Y \subsetneq Y^{\prime} .
\end{aligned}
$$

Then, writing $v=p(Y) u$ and $c_{\mathcal{F}}=c_{\mathcal{F}_{1}} \times c_{\mathcal{F}_{2}}$, we have

$$
\pi_{\mathcal{F}}(X)=\pi_{\mathcal{F}_{1}}(-Y) \pi_{\mathcal{F}_{2}}\left(Y^{\prime}\right) \prod_{x_{i} \in Y, x_{j} \in Y^{\prime}} \frac{1-q^{2} x_{i}^{-1} x_{j}}{1-x_{i}^{-1} x_{j}},
$$

and $\mathcal{A}_{\mathcal{F}}(X, u)(1-p(Y) u)$ is equal to

$$
\begin{aligned}
& \mathcal{A}_{\mathcal{F}_{1}}(-Y, v) \mathcal{A}_{\mathcal{F}_{2}}\left(Y^{\prime}, v\right)(1-v)\left(\frac{v}{1-v}+\frac{\chi(Y=X)}{(-q)_{\left|Y \backslash F_{t-1}\right|}}\right) \\
& \quad \times\left(\frac{v}{1-v}+\frac{1}{(-q)_{\left|Y \backslash F_{t-1}\right|}}\right)^{-1}\left(\frac{v}{1-v}+\frac{1}{(-q)_{\left|F_{t+1} \backslash Y\right|}}\right)^{-1} .
\end{aligned}
$$


Thus when $u=p(-Y)$, i.e., $v=1$,

$$
\begin{aligned}
& {\left[\pi_{\mathcal{F}}(X) \mathcal{A}_{\mathcal{F}}(X, u)(1-p(Y) u)\right]_{u=p(-Y)}=} {\left[\pi_{\mathcal{F}_{1}}(-Y) \mathcal{A}_{\mathcal{F}_{1}}(-Y, v)(1-v) \pi_{\mathcal{F}_{2}}\left(Y^{\prime}\right) \mathcal{A}_{\mathcal{F}_{2}}\left(Y^{\prime}, v\right)\right.} \\
&\times(1-v)]_{v=1} \prod_{x_{i} \in Y, x_{j} \in Y^{\prime}} \frac{1-q^{2} x_{i}^{-1} x_{j}}{1-x_{i}^{-1} x_{j}} .
\end{aligned}
$$

Using (35) and the result of $c(\emptyset)$, which can be written

$$
\left[\sum_{\mathcal{F}} \pi_{\mathcal{F}} \mathcal{A}_{\mathcal{F}}(X, u)(1-u)\right]_{u=1}=\Phi(X),
$$

we get

$$
c(Y)=\Phi(-Y) \Phi\left(Y^{\prime}\right) \prod_{x_{i} \in Y, x_{j} \in Y^{\prime}} \frac{1-q^{2} x_{i}^{-1} x_{j}}{1-x_{i}^{-1} x_{j}} .
$$

Each subset $Y$ of $X$ can be encoded by a sequence $\xi \in\{ \pm 1\}^{n}$ according to the rule: $\xi_{i}=1$ if $x_{i} \notin Y$ and $\xi_{i}=-1$ if $x_{i} \in Y$. Hence

$$
c(Y)=\Phi\left(X^{\xi}\right) .
$$

Note also that

$$
p(Y)=\prod_{i} x_{i}^{\left(1-\xi_{i}\right) / 2}, \quad p(-Y)=\prod_{i} x_{i}^{\left(\xi_{i}-1\right) / 2} .
$$

Now, extracting the coefficients of $u^{k}$ in the equation:

$$
S(u)=\sum_{Y \subseteq X} \frac{c(Y)}{1-p(Y) u},
$$

yields

$$
\sum_{\lambda_{1} \leq k}\left(\prod_{i=1}^{k-1}(-q)_{m_{i}}\right) P_{\lambda}\left(X, q^{2}\right)=\sum_{Y \subseteq X} c(Y) p(Y)^{k} .
$$

Finally, substituting the value of $c(Y)$ in the above formula we obtain (5).

\subsection{Proof of Corollary 1}

Recall [9, p. 213] that if $x_{i}=z q^{2 i-2}(1 \leq i \leq n)$ then:

$$
P_{\lambda^{\prime}}\left(X, q^{2}\right)=z^{|\lambda|} q^{2 n(\lambda)}\left[\begin{array}{l}
n \\
\lambda
\end{array}\right]_{q^{2}} .
$$


Replacing each partition $\lambda$ by its conjugate $\lambda^{\prime}$ on the left-hand side of (5) yields the left-hand side of (6). Set

$$
\Psi(X)=\prod_{i} \frac{1}{1-x_{i}^{2}} \prod_{j<k} \frac{1-q^{2} x_{j} x_{k}}{1-x_{j} x_{k}} .
$$

Then, for any $\xi \in\{ \pm 1\}^{n}$ such that the number of $\xi_{i}=-1$ is $r, 0 \leq r \leq n$, we can write $\Phi\left(X^{\xi}\right)$ as follows:

$$
\Phi\left(X^{\xi}\right)=\Psi\left(X^{\xi}\right) \prod_{i} \frac{1+q x_{i}^{\xi_{i}}}{1-x_{i}^{\xi_{i}}}\left(1-x_{i}^{2 \xi_{i}}\right)
$$

which is readily seen to equal 0 unless $\xi \in\{-1\}^{r} \times\{1\}^{n-r}$. Now, in the latter case, we have $\prod_{i} x_{i}^{k\left(1-\xi_{i}\right) / 2}=z^{k r} q^{2 k\left(\begin{array}{c}r \\ 2\end{array}\right)}$,

$$
\prod_{i=1}^{n} \frac{1+q x_{i}^{\xi_{i}}}{1-x_{i}^{\xi_{i}}}\left(1-x_{i}^{2 \xi_{i}}\right)=\frac{\left(z^{2} ; q^{4}\right)_{n}}{z^{2 r} q^{4\left(\begin{array}{c}
r \\
2
\end{array}\right)-r}} \frac{\left(-z / q ; q^{2}\right)_{r}}{\left(z ; q^{2}\right)_{r}} \frac{\left(-z q^{2 r+1} ; q^{2}\right)_{n-r}}{\left(z q^{2 r} ; q^{2}\right)_{n-r}}
$$

and [12, p. 476]:

$$
\Psi\left(X^{\xi}\right)=(-1)^{r} z^{2 r} q^{6\left(\begin{array}{c}
r \\
2
\end{array}\right)}\left[\begin{array}{l}
n \\
r
\end{array}\right] \frac{1-z^{2} q^{4 r-2}}{\left(z q^{r-1}\right)_{n+1}} .
$$

Substituting these into the right-hand side of (5) we obtain the right-hand side of (6) after simple manipulations.

When $n \rightarrow+\infty$, since $\left[\begin{array}{l}n \\ \lambda\end{array}\right] \rightarrow \frac{1}{(q)_{\lambda}}$, equation (6) reduces to:

$$
\sum_{l(\lambda) \leq k} \frac{z^{|\lambda|} q^{2 n(\lambda)}}{(-q)_{\lambda_{k}}(q)_{\lambda}}=(-z / q)_{\infty} \sum_{r \geq 0} \frac{(-1)^{r} z^{k r} q^{r+(2 k+2)\left(\begin{array}{c}
r \\
2
\end{array}\right)}}{\left(q^{2} ; q^{2}\right)_{r}\left(z^{2} q^{2 r-2}\right)_{\infty}}\left(1-z q^{2 r-1}\right) .
$$

Furthermore, as in Section 2, setting $z=q^{2}$ and $z=q$ in (40) yields (17) and (21), respectively.

\section{Elementary approach and proof of Theorem 2}

\subsection{Preliminaries}

We will need the following result, which corresponds to the case $k \rightarrow \infty$ in (6), and can be proved in an elementary way:

Lemma 1. For $n \geq 0$

$$
\sum_{\lambda} z^{|\lambda|} q^{2 n(\lambda)}(-q)_{\lambda}\left[\begin{array}{l}
n \\
\lambda
\end{array}\right]_{q^{2}}=\frac{(-z)_{2 n}}{\left(z^{2} ; q^{2}\right)_{n}}
$$


Proof: Recall the following identity, which is proved in [7]:

$$
q^{\left(\begin{array}{c}
m \\
2
\end{array}\right)+n(\mu)}\left[\begin{array}{l}
n \\
m
\end{array}\right]\left[\begin{array}{l}
n \\
\mu
\end{array}\right]=\sum_{\lambda} q^{n(\lambda)}\left[\begin{array}{l}
n \\
\lambda
\end{array}\right] \prod_{i \geq 1}\left[\begin{array}{c}
\lambda_{i}-\lambda_{i+1} \\
\lambda_{i}-\mu_{i}
\end{array}\right],
$$

where the sum is over all partitions $\lambda$ such that $\lambda / \mu$ is a horizontal $m$-strip, i.e., $\mu \subseteq \lambda$, $|\lambda / \mu|=m$ and there is at most one cell in each column of the Ferrers diagram of $\lambda / \mu$.

We also need

$$
\sum_{\lambda} z^{|\lambda|} q^{n(\lambda)}\left[\begin{array}{l}
n \\
\lambda
\end{array}\right]=\frac{(-z)_{n}}{\left(z^{2}\right)_{n}}
$$

which can be found in $[7,12]$.

Using (43) with $q$ replaced by $q^{2}$ and (2), the right-hand side of (41) can be written

$$
\begin{aligned}
\frac{\left(-z ; q^{2}\right)_{n}}{\left(z^{2} ; q^{2}\right)_{n}}\left(-z q ; q^{2}\right)_{n} & =\sum_{\mu, m} z^{|\mu|} q^{2 n(\mu)}\left[\begin{array}{l}
n \\
\mu
\end{array}\right]_{q^{2}} z^{m} q^{2\left(\begin{array}{c}
m \\
2
\end{array}\right)+m}\left[\begin{array}{c}
n \\
m
\end{array}\right]_{q^{2}} \\
& =\sum_{\lambda, m} z^{|\lambda|} q^{2 n(\lambda)}\left[\begin{array}{l}
n \\
\lambda
\end{array}\right]_{q^{2}} \prod_{i \geq 1} \sum_{r_{i} \geq 0} q^{r_{i}}\left[\begin{array}{c}
\lambda_{i}-\lambda_{i+1} \\
r_{i}
\end{array}\right]_{q^{2}},
\end{aligned}
$$

where the last equality follows from (42), setting $r_{i}=\lambda_{i}-\mu_{i}$ for $i \geq 1$. Now we conclude by using (10).

Recall the following extension of the $n \rightarrow \infty$ case of (41), which is Stembridge's lemma 3.3 (b) in [12], and identity (60) in [7]:

$$
\sum_{\lambda} z^{|\lambda|} q^{2 n(\lambda)} \frac{\left(a, b ; q^{-1}\right)_{\lambda_{1}}}{(q)_{\lambda}}=\frac{(a z, b z)_{\infty}}{(z, a b z)_{\infty}} .
$$

Now, using (41), we are able to prove directly identity (6) in Corollary 1, and then using (44), to deduce an elementary proof of (7) in Theorem 2.

\subsection{Elementary proof of Corollary 1}

Consider the generating function of the left-hand side of (6):

$$
\begin{aligned}
\varphi(u) & =\sum_{k \geq 0} u^{k} \sum_{l(\lambda) \leq k} \frac{(-q)_{\lambda}}{(-q)_{\lambda_{k}}} z^{|\lambda|} q^{2 n(\lambda)}\left[\begin{array}{l}
n \\
\lambda
\end{array}\right]_{q^{2}} \\
& =\sum_{\lambda} u^{l(\lambda)} z^{|\lambda|} q^{2 n(\lambda)}(-q)_{\lambda}\left[\begin{array}{l}
n \\
\lambda
\end{array}\right]_{q^{2}} \sum_{k \geq 0} \frac{u^{k}}{(-q)_{\lambda_{k+l(\lambda)}}} \\
& =\sum_{\lambda} u^{l(\lambda)} z^{|\lambda|} q^{2 n(\lambda)}(-q)_{\lambda}\left[\begin{array}{l}
n \\
\lambda
\end{array}\right]_{q^{2}}\left(\frac{u}{1-u}+\frac{1}{(-q)_{\lambda_{l(\lambda)}}}\right),
\end{aligned}
$$


where the last equality follows from the fact that $\lambda_{k+l(\lambda)}=0$ unless $k=0$. Now, each partition $\lambda$ with parts bounded by $n$ can be encoded by a pair of sequences $v=\left(v_{0}, v_{1}, \ldots, v_{l}\right)$ and $\mathbf{m}=\left(m_{0}, \ldots, m_{l}\right)$ such that $\lambda=\left(v_{0}^{m_{0}}, \ldots, v_{l}^{m_{l}}\right)$, where $n=v_{0}>v_{1}>\cdots>v_{l}>0$ and $v_{i}$ has multiplicity $m_{i} \geq 1$ for $1 \leq i \leq l$ and $v_{0}=n$ has multiplicity $m_{0} \geq 0$. Using the notation:

$$
\langle\alpha\rangle=\frac{\alpha}{1-\alpha}, \quad u_{i}=z^{i} q^{i(i-1)} \quad \text { for } \quad i \geq 0,
$$

we can then rewrite (46) as follows:

$$
\begin{aligned}
\varphi(u)= & \sum_{\nu}(-q)_{v}\left[\begin{array}{l}
n \\
v
\end{array}\right]_{q^{2}}\left(\langle u\rangle+\frac{1}{(-q)_{v_{l}}}\right) \\
& \times \sum_{\mathbf{m}}\left(\left(u_{n} u\right)^{m_{0}}+\frac{\chi\left(m_{0}=0\right)}{(-q)_{n-v_{1}}}\right) \prod_{i=1}^{l}\left(u_{v_{i}} u\right)^{m_{i}} \\
= & \sum_{v} \frac{\left(q^{2} ; q^{2}\right)_{n}}{(q)_{v}} B_{v},
\end{aligned}
$$

where the sum is over all strict partitions $v=\left(v_{0}, v_{1}, \ldots, v_{l}\right)$ and

$$
B_{v}=\left(\langle u\rangle+\frac{1}{(-q)_{v_{l}}}\right)\left(\left\langle u_{r} u\right\rangle+\frac{1}{(-q)_{n-v_{1}}}\right) \prod_{i=1}^{l}\left\langle u_{v_{i}} u\right\rangle .
$$

So $\varphi(u)$ is a rational fraction with simple poles at $u_{r}^{-1}$ for $0 \leq r \leq n$. Let $b_{r}(z, n)$ be the corresponding residue of $\varphi(u)$ at $u_{r}^{-1}$ for $0 \leq r \leq n$. Then, it follows from (47) that

$$
b_{r}(z, n)=\sum_{v} \frac{\left(q^{2} ; q^{2}\right)_{n}}{(q)_{v}}\left[B_{v}\left(1-u_{r} u\right)\right]_{u=u_{r}^{-1}} .
$$

We shall first consider the cases where $r=0$ or $n$. Using (46) and (41) we have

$$
b_{0}(z, n)=[\varphi(u)(1-u)]_{u=1}=\frac{(-z)_{2 n}}{\left(z^{2} ; q^{2}\right)_{n}} .
$$

Now, by (47) and (48) we have

$$
b_{0}(z, n)=\sum_{v} \frac{\left(q^{2} ; q^{2}\right)_{n}}{(q)_{v}}\left(\left\langle u_{n}\right\rangle+\frac{1}{(-q)_{n-v_{1}}}\right) \prod_{i=1}^{l}\left\langle u_{v_{i}}\right\rangle,
$$

and

$$
b_{n}(z, n)=\sum_{\nu} \frac{\left(q^{2} ; q^{2}\right)_{n}}{(q)_{v}}\left(\left\langle 1 / u_{n}\right\rangle+\frac{1}{(-q)_{v_{l}}}\right) \prod_{i=1}^{l}\left\langle u_{v_{i}} / u_{n}\right\rangle,
$$


which, by setting $\mu_{i}=n-v_{l+1-i}$ for $1 \leq i \leq l$ and $\mu_{0}=n$, can be written as

$$
b_{n}(z, n)=\sum_{\mu} \frac{\left(q^{2} ; q^{2}\right)_{n}}{(q)_{\mu}}\left(\left\langle 1 / u_{n}\right\rangle+\frac{1}{(-q)_{n-\mu_{1}}}\right) \prod_{i=1}^{l}\left\langle u_{n-\mu_{i}} / u_{n}\right\rangle .
$$

Comparing (52) with (50) we see that $b_{n}(z, n)$ is equal to $b_{0}(z, n)$ with $z$ replaced by $z^{-1} q^{-2 n+2}$. It follows from (49) that

$$
b_{n}(z, n)=b_{0}\left(z^{-1} q^{-2 n+2}, n\right)=(-1)^{n} q^{n^{2}} \frac{(-z / q)_{2 n}}{\left(z^{2} q^{2 n-2} ; q^{2}\right)_{n}} .
$$

Consider now the case where $0<r<n$. Clearly, for each partition $v$, the corresponding summand in (48) is not zero only if $v_{j}=r$ for some $j, 0 \leq j \leq n$. Furthermore, each such partition $v$ can be split into two strict partitions $\rho=\left(\rho_{0}, \rho_{1}, \ldots, \rho_{j-1}\right)$ and $\sigma=\left(\sigma_{0}, \ldots, \sigma_{l-j}\right)$ such that $\rho_{i}=v_{i}-r$ for $0 \leq i \leq j-1$ and $\sigma_{s}=v_{j+s}$ for $0 \leq s \leq l-j$. So we can write (48) as follows:

$$
b_{r}(z, n)=\left[\begin{array}{l}
n \\
r
\end{array}\right]_{q^{2}} \sum_{\rho} \frac{\left(q^{2} ; q^{2}\right)_{n-r}}{(q)_{\rho}} F_{\rho}(r) \times \sum_{\sigma} \frac{\left(q^{2} ; q^{2}\right)_{r}}{(q)_{\sigma}} G_{\sigma}(r)
$$

where for $\rho=\left(\rho_{0}, \rho_{1}, \ldots, \rho_{l}\right)$ with $\rho_{0}=n-r$,

$$
F_{\rho}(r)=\left(\left\langle u_{n} / u_{r}\right\rangle+\frac{1}{(-q)_{n-r-\rho_{1}}}\right) \prod_{i=1}^{l(\rho)}\left\langle u_{\rho_{i}+r} / u_{r}\right\rangle
$$

and for $\sigma=\left(\sigma_{0}, \ldots, \sigma_{l}\right)$ with $\sigma_{0}=n$,

$$
G_{\sigma}(r)=\left(\left\langle 1 / u_{r}\right\rangle+\frac{1}{(-q)_{\sigma_{l}}}\right) \prod_{i=1}^{l(\sigma)}\left\langle u_{\sigma_{i}} / u_{r}\right\rangle
$$

Comparing with (50) and (52) and using (49) and (53) we obtain

$$
\begin{aligned}
b_{r}(z, n) & =\left[\begin{array}{l}
n \\
r
\end{array}\right]_{q^{2}} b_{0}\left(z q^{2 r}, n-r\right) b_{r}(z, r) \\
& =(-1)^{r} q^{r+2\left(\begin{array}{l}
n \\
r
\end{array}\right)} \frac{(-z / q)_{2 n+1}}{\left(z^{2} q^{2 r-2}, q^{2}\right)_{n+1}}\left(1-z q^{4 r-1}\right) .
\end{aligned}
$$

Finally, extracting the coefficients of $u^{k}$ in the equation

$$
\varphi(u)=\sum_{p=0}^{n} \frac{b_{r}(z, n)}{1-u_{r} u}
$$

and using the values for $b_{r}(z, n)$ we obtain (6). 


\subsection{Proof of Theorem 2}

Consider the generating function of the left-hand side of (7):

$$
\begin{aligned}
\varphi_{a b}(u) & :=\sum_{k \geq 0} u^{k} \sum_{l(\lambda) \leq k} z^{|\lambda|} q^{2 n(\lambda)} \frac{\left(a, b ; q^{-1}\right)_{\lambda_{1}}}{(q)_{\lambda}(-q)_{\lambda_{k}}} \\
& =\sum_{\lambda} \sum_{k \geq 0} u^{k+l(\lambda)} z^{|\lambda|} q^{2 n(\lambda)} \frac{\left(a, b ; q^{-1}\right)_{\lambda_{1}}}{(q)_{\lambda}(-q)_{\lambda_{l(\lambda)+k}}},
\end{aligned}
$$

where the sum is over all the partitions $\lambda$, and as before $\lambda_{l(\lambda)+k}=0$ unless $k=0$. Thus

$$
\varphi_{a b}(u)=\sum_{\lambda} u^{l(\lambda)} z^{|\lambda|} q^{2 n(\lambda)} \frac{\left(a, b ; q^{-1}\right)_{\lambda_{1}}}{(q)_{\lambda}}\left(\frac{u}{1-u}+\frac{1}{(-q)_{\lambda_{l(\lambda)}}}\right) .
$$

As in the elementary proof of Corollary 1 , we can replace any partition $\lambda$ by a pair $(\nu, \mathbf{m})$, where $v$ is a strict partition consisting of distinct parts $v_{1}, \ldots, v_{l}$ of $\lambda$, so that $v_{1}>\cdots>$ $v_{l}>0$, and $\mathbf{m}=\left(m_{1}, \ldots, m_{l}\right)$ is the sequence of multiplicities of $v_{i}$ for $1 \leq i \leq l$. Therefore

$$
\begin{aligned}
\varphi_{a b}(u) & =\sum_{\nu, \mathbf{m}} \frac{\left(a, b ; q^{-1}\right)_{v_{1}}}{(q)_{v}}\left(\frac{u}{1-u}+\frac{1}{(-q)_{v_{l}}}\right) \prod_{i=1}^{l}\left(u_{v_{i}} u\right)^{m_{i}} \\
& =\sum_{\nu} \frac{\left(a, b ; q^{-1}\right)_{\nu_{1}}}{(q)_{v}}\left(\langle u\rangle+\frac{1}{(-q)_{v_{l}}}\right) \prod_{i=1}^{l}\left\langle u_{v_{i}} u\right\rangle,
\end{aligned}
$$

where the sum is over all the strict partitions $v$. Each of the terms in this sum, as a rational function of $u$, has a finite set of simple poles, which may occur at the points $u_{r}^{-1}$ for $r \geq 0$. Therefore, each term is a linear combination of partial fractions. Moreover, the sum of their expansions converges coefficientwise. So $\varphi_{a b}$ has an expansion

$$
\varphi_{a b}(u)=\sum_{r \geq 0} \frac{c_{r}}{1-u z^{r} q^{r(r-1)}}
$$

where $c_{r}$ denotes the formal sum of partial fraction coefficients contributed by the terms of (55). It remains to compute these residues $c_{r}(r \geq 0)$. By using (44) and (55), we get immediately

$$
c_{0}=\left[\varphi_{a b}(u)(1-u)\right]_{u=1}=\frac{(a z, b z)_{\infty}}{(z, a b z)_{\infty}} .
$$

In view of (55), this yields the identity

$$
\sum_{v} \frac{\left(a, b ; q^{-1}\right)_{v_{1}}}{(q)_{v}} \prod_{i=1}^{l}\left\langle u_{v_{i}}\right\rangle=\frac{(a z, b z)_{\infty}}{(z, a b z)_{\infty}} .
$$

Clearly, a summand in (55) has a non zero contribution to $c_{r}(r>0)$ only if the corresponding partition $v$ has a part equal to $r$. For any partition $v$ such that $\exists j \mid v_{j}=r$, set 
$\rho_{i}:=v_{i}-r$ for $1 \leq i<j$ and $\sigma_{i}:=v_{i+j}$ for $0 \leq i \leq l-j$, we then get two partitions $\rho$ and $\sigma$, with $\sigma_{i}$ bounded by $r$. Multiplying (55) by $\left(1-u_{r} u\right)$ and setting $u=1 / u_{r}$ we obtain

$$
\begin{aligned}
c_{r}= & \sum_{\rho} \frac{\left(a, b ; q^{-1}\right)_{\rho_{1}+r}}{(q)_{\rho}} \prod_{i=1}^{j-1}\left\langle u_{r+\rho_{i}} / u_{r}\right\rangle \\
& \times \sum_{\sigma} \frac{1}{(q)_{\sigma}}\left(\left\langle 1 / u_{r}\right\rangle+\frac{1}{(-q)_{\sigma_{l-j}}}\right) \prod_{i=1}^{l-j}\left\langle u_{\sigma_{i}} / u_{r}\right\rangle .
\end{aligned}
$$

In view of (51) the inner sum over $\sigma$ is equal to $b_{r}(z, r) /\left(q^{2}, q^{2}\right)_{r}$, and applying (53) we get

$$
\begin{aligned}
& c_{r}=(-1)^{r} q^{r+2\left(\begin{array}{c}
r \\
2
\end{array}\right)} \frac{(-z / q)_{2 r}}{\left(z^{2} q^{2 r-2}, q^{2}\right)_{r}} \frac{\left(a, b ; q^{-1}\right)_{r}}{\left(q^{2} ; q^{2}\right)_{r}} \\
& \times \sum_{\rho} \frac{\left(a q^{-r}, b q^{-r} ; q^{-1}\right)_{\rho_{1}}}{(q)_{\rho}} \prod_{i=1}^{j-1}\left\langle u_{r+\rho_{i}} / u_{r}\right\rangle \text {. }
\end{aligned}
$$

Now, the sum over $\rho$ can be computed using (56) with $a, b$ and $z$ replaced by $a q^{-r}, b q^{-r}$ and $z q^{2 r}$ respectively. After simplification, we obtain

$$
c_{r}=(-1)^{r} q^{r+2\left(\begin{array}{c}
r \\
2
\end{array}\right)} \frac{(-z / q)_{\infty}}{\left(z^{2} q^{2 r-2}, q^{2}\right)_{\infty}} \frac{\left(a, b ; q^{-1}\right)_{r}}{\left(q^{2} ; q^{2}\right)_{r}} \frac{\left(a z q^{r}, b z q^{r}\right)_{\infty}}{(a b z)_{\infty}},
$$

which completes the proof.

Acknowledgments The second and third authors are partially supported by EC's IHRP Programme, within the Research Training Network "Algebraic Combinatorics in Europe", grant HPRN-CT-2001-00272.

\section{References}

1. G.E. Andrews, The theory of partitions, Encyclopedia of mathematics and its applications, AddisonWesley, Reading, Massachusetts, 2 (1976).

2. G.E. Andrews, Multiple series Rogers-Ramanujan type identities, Pacific J. Math., 114(2) (1984), 267283.

3. D.M. Bressoud, An analytic generalization of the Rogers-Ramanujan identities with interpretation, Quart. J. Math. Oxford Ser. (2), 31 (124) (1980), 385-399.

4. D.M. Bressoud, Analytic and combinatorial generalizations of the Rogers-Ramanujan identities, Mem. Amer. Math. Soc., 24 (227) (1980).

5. G. Gasper and M. Rahman, Basic Hypergeometric Series, 2nd edn., Encyclopedia of Mathematics and its Applications 96, Cambridge University Press, Cambridge, 2004.

6. F. Jouhet and J. Zeng, Some new identities for Schur functions, Adv. Appl. Math., 27 (2001), $493-509$.

7. F. Jouhet and J. Zeng, New Identities of Hall-Littlewood Polynomials and Applications, The Ramanujan J. 10 (2005), 89-112.

8. N. Kawanaka, A q-series identity involving Schur functions and related topics, Osaka J. Math., 36 (1999), $157-176$

9. I.G. Macdonald, Symmetric functions and Hall polynomials, Clarendon Press, second edition, Oxford, 1995. 
10. L.J. Slater, A new proof of Rogers's transformations of infinite series, Proc. London Math. Soc., 53(2) (1951), 460-475.

11. L.J. Slater, Further identities of the Rogers-Ramanujan Type, Proc. London Math. Soc., 54(2) (1951-52), $147-167$.

12. J.R. Stembridge, Hall-Littlewood functions, plane partitions, and the Rogers-Ramanujan identities, Trans. Amer. Math. Soc., 319(2) (1990), 469-498.

13. J. Zeng, On the $q$-variations of Sylvester's bijection, The Ramanujan J. 9 (2005), 289-303. 